\title{
Korean Guidelines for Diagnosis and Management of Interstitial Lung Diseases: Part 3. Idiopathic Nonspecific Interstitial Pneumonia
}

\author{
Jongmin Lee, M.D. ${ }^{1}{ }^{\mathbb{D}}$, Yong Hyun Kim, M.D. ${ }^{2}{ }^{\mathbb{D}}$, Ji Young Kang, M.D., Ph.D. ${ }^{1}$, \\ Yangjin Jegal, M.D., Ph.D. ${ }^{3}$ and So Young Park, M.D., Ph.D. ${ }^{4}$, on behalf of Korean Interstitial Lung \\ Diseases Study Group \\ ${ }^{1}$ Division of Pulmonology and Allergy, Department of Internal Medicine, Seoul St. Mary's Hospital, College of Medicine, The \\ Catholic University School of Medicine, Seoul, ${ }^{2}$ Division of Allergy and Pulmonology, Department of Internal Medicine, \\ Bucheon St. Mary's Hospital, The Catholic University School of Medicine, Bucheon, ${ }^{3}$ Division of Pulmonary and Critical \\ Care Medicine, Department of Internal Medicine, Ulsan University Hospital, Ulsan University College of Medicine, Ulsan, \\ ${ }^{4}$ Department of Pulmonary and Critical Care Medicine, Chungnam National University Hospital, Daejeon, Korea
}

Idiopathic nonspecific interstitial pneumonia (NSIP) is one of the varieties of idiopathic interstitial pneumonias. Diagnosis of idiopathic NSIP can be done via multidisciplinary approach in which the clinical, radiologic, and pathologic findings were discussed together and exclude other causes. Clinical manifestations include subacute or chronic dyspnea and cough that last an average of 6 months, most of which occur in non-smoking, middle-aged women. The common findings in thoracic high-resolution computed tomography in NSIP are bilateral reticular opacities, traction bronchiectasis, reduced volume of the lobes, and ground-glass opacity in the lower lungs. These lesions can involve diffuse bilateral lungs or subpleural area. Unlike usual interstitial pneumonia, honeycombing is sparse or absent. Pathology shows diffuse interstitial inflammation and fibrosis which are temporally homogeneous, namely NSIP pattern. Idiopathic NSIP is usually treated with steroid only or combination with immunosuppressive agents such as azathioprine, cyclophosphamide, cyclosporine, and mycophenolate mofetil. Prognosis of idiopathic NSIP is better than idiopathic pulmonary fibrosis. Many studies have reported a 5 -year survival rate of more than $70 \%$.

Keywords: Lung Diseases, Interstitial; Idiopathic Interstitial Pneumonias; Guideline

Address for correspondence: Yong Hyun Kim, M.D.

Division of Allergy and Pulmonology, Department of Internal Medicine, Bucheon St. Mary's Hospital, College of Medicine, The Catholic University of Korea, 327 Sosa-ro, Wonmi-gu, Bucheon 14647, Korea

Phone: 82-32-340-7039, Fax: 82-32-340-2669

E-mail:kyh30med@catholic.ac.kr

Received: Dec. 21, 2018

Revised: Apr. 1, 2019

Accepted: Apr. 23, 2019

Published online: May. 31, 2019

(9) It is identical to the Creative Commons Attribution Non-Commercial License (http://creativecommons.org/licenses/by-nc/4.0/).

\section{Introduction}

Idiopathic nonspecific interstitial pneumonia (NSIP) is one of the varieties of idiopathic interstitial pneumonias (IIP). NSIP used to be considered as not an independent disease entity but provisional diagnosis. However, now, it is recognized as a distinct entity with clinical features that differentiate it from other interstitial pneumonias ${ }^{1,2}$. Diagnosis of idiopathic NSIP can be done via multidisciplinary approach in which the clinical, radiologic and pathologic findings are discussed together and other causes are excluded. 


\section{History and Epidemiology}

\section{History}

NSIP began to gain attention in 1994 when Katzenstein and Fiorelli ${ }^{3}$ described histologic findings that did not fit the traditional Liebow classification of interstitial pneumonia. These pathology showed temporally uniform inflammation and fibrosis which distinguished them from other interstitial pneumonias, such as usual interstitial pneumonia (UIP). However, authors at the time concluded that it would be unreasonable to regard NSIP as an independent disease entity. In subsequent studies, the possibility was raised that NSIP might be independent disease entity although the histologic findings of NSIP were associated with a variety of causes including connective tissue disease (CTD) ${ }^{2}$. And, at the 2002 American Thoracic Society (ATS)/European Respiratory Society (ERS) international consensus classification, NSIP was classified as "provisional type" interstitial pneumonia ${ }^{4}$. In 2008, Travis et al. ${ }^{2}$ reported that idiopathic NSIP is a distinct clinical entity that is distinguishable from other interstitial pneumonias. They also reported that NSIP occurred mostly in non-smoking middle aged women, and its prognosis is good. In the recent revised ATS/ERS international consensus classification, idiopathic NSIP was classified as a distinct entity among the IIPs ${ }^{1}$.

\section{Epidemiology}

The incidence and prevalence of NSIP are not clearly known. However, according to several retrospective cohort studies, the prevalence has been estimated at 1 to 9 per 100,000 people $^{5}$, and the incidence has been estimated at around 3 per one million people ${ }^{6}$. According to a nationwide survey conducted by the Korean Academy and Tuberculosis and Respiratory Diseases in 2008, NSIP was the second common IIP next to idiopathic pulmonary fibrosis (IPF), accounting for $11.9 \%$ of 2,186 patients with $\mathrm{IIP}^{7}$. In a recent cohort of a university hospital in Denmark, 431 cases of interstitial lung diseases were analyzed from 2003 to 2009. NSIP accounted for $7 \%$ of them. And it was the fourth most common interstitial lung disease following IPF, CTD interstitial pneumonia, and hypersensitivity pneumonitis (HP) ${ }^{6}$. NSIP is more common in females and non-smokers than their counterparts, and occurs at lower ages than $\mathrm{IPF}^{2,7}$.

\section{Clinical Features and Diagnosis}

\section{Clinical features}

Clinical manifestations include subacute or chronic dyspnea and cough that last an average of 6 months, most of which occur in non-smoking, middle-aged women. Bilateral end-inspiratory crackles at the base of chest can be heard but most of physical findings are nonspecific. Pulmonary function tests show a restrictive ventilatory defect ${ }^{2}$. Because similar clinical manifestations can occur in the patients with HP, drug toxicities and occupational lung diseases, detailed history taking is necessary to find exposure to specific antigens such as birds, drugs, or occupational exposures. In addition, the NSIP pattern is known to be the most common histologic pattern of pulmonary involvement in various CTDs. Therefore, in order to exclude specific CTDs, it is important to check for symptoms such as Raynaud's phenomenon, arthralgia or arthritis, skin rash, dry mouth, and dry eye. Autoantibodies, such as anti-nuclear antibody, rheumatoid factor, and anti-cyclic citrullinated peptide, are also useful markers and should be considered for exclude CTDs. However, in many patients, even if autoantibody is positive, clinical manifestations are not sufficient for diagnosis of specific CTD ${ }^{2,9-12}$. If the diagnostic criteria of a specific CTD are not met despite autoantibodies or some clinical features are similar to those of CTD, diagnosis as an idiopathic NSIP can be made. Recently, there has been a proposal to use the term "interstitial pneumonia with autoimmune features (IPAF)." IPAF is a term to characterize the heterogenous group of patients with IIPs who have a clinical flavor of underlying CTDs but do not meet the current American College of Rheumatology criteria for CTDs ${ }^{13}$. But, the concepts of IPAF are proposed to provide a platform for the prospective study of these heterogenous group and are not intended as guidelines for clinical care. At present, IPAF will need to be validated via prospective research studies and we should be careful to apply it to real clinical field.

\section{Diagnosis}

The diagnosis of NSIP is similar to that of other interstitial pneumonias including IPF, and it is important to diagnose it

Table 1. Differential diagnosis for idiopathic nonspecific interstitial pneumonia

Interstitial lung disease associated or related with connective
tissue disease
Hypersensitivity pneumonitis
Other idiopathic interstitial pneumonia (organizing pneumonia,
idiopathic pulmonary fibrosis, smoking-related interstitial
pneumonia, etc.)
Drug-related pneumonitis (flecainide, amiodarone,
methotrexate, nitrofurantoin, etc.)
Infection (Pneumocystis carinii, human immunodeficiency virus,
etc.)
Chronic eosinophilic pneumonia
Lymphoproliferative lung disease (lymphoma, lymphoid
interstitial pneumonia)


through multidisciplinary diagnosis (MDD) with respiratory physicians, radiologists, and pathologists. MDD is particularly important to diagnose idiopathic NSIP because NSIP can occur in various clinical diseases including HP, CTD, and drug reactions ${ }^{1}$. The differential diagnosis is summarized in Table 1.

\section{1) Radiological examination}

The common findings in thoracic high-resolution computed tomography (HRCT) in NSIP are bilateral reticular opacities, traction bronchiectasis, reduced volume of the lobes, and ground-glass opacity in the lower lungs ${ }^{2,14-16}$. These lesions can involve diffuse bilateral lungs or subpleural area. Although it is not common, $20 \%$ of patients showed subpleural sparing which is helpful in distinguishing NSIP from IPF. Unlike UIP, honeycombing is sparse or absent (Figure 1). Consolidation, if present, indicates organizing pneumonia component and may suggest the presence of CTD ${ }^{2}$. Unlike UIP, it is not possible to diagnose NSIP with HRCT findings alone.

\section{2) Bronchoscopy}

Although T lymphocyte fractions increase $(>20 \%)$ in the bronchoalveolar lavage (BAL) fluid of most patients, it is nonspecific finding and only plays an auxiliary role for differential diagnosis. For example, increase of $\mathrm{T}$ lymphocyte fractions in

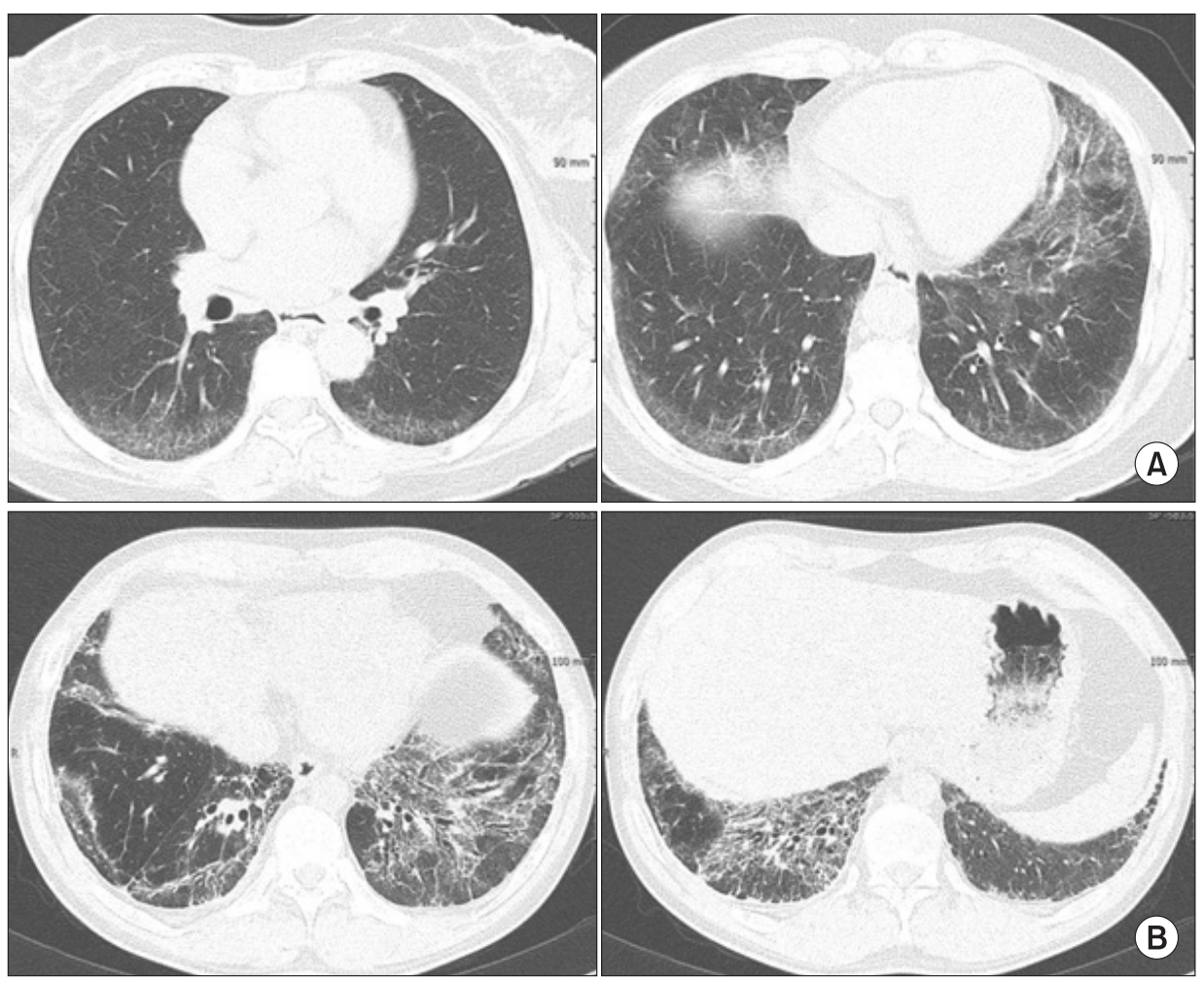

Figure 1. High-resolution computed tomography (HRCT) of nonspecific interstitial pneumonia. (A) HRCT axial images (lung window setting) show ill-defined ground glass opacities in periphery of the both lower lobes. (B) HRCT axial images (lung window setting) at level of both lower lobes demonstrate reticulation, illdefined ground glass opacities, and traction bronchiectasis along bronchovascular bundles or along subpleural lungs.
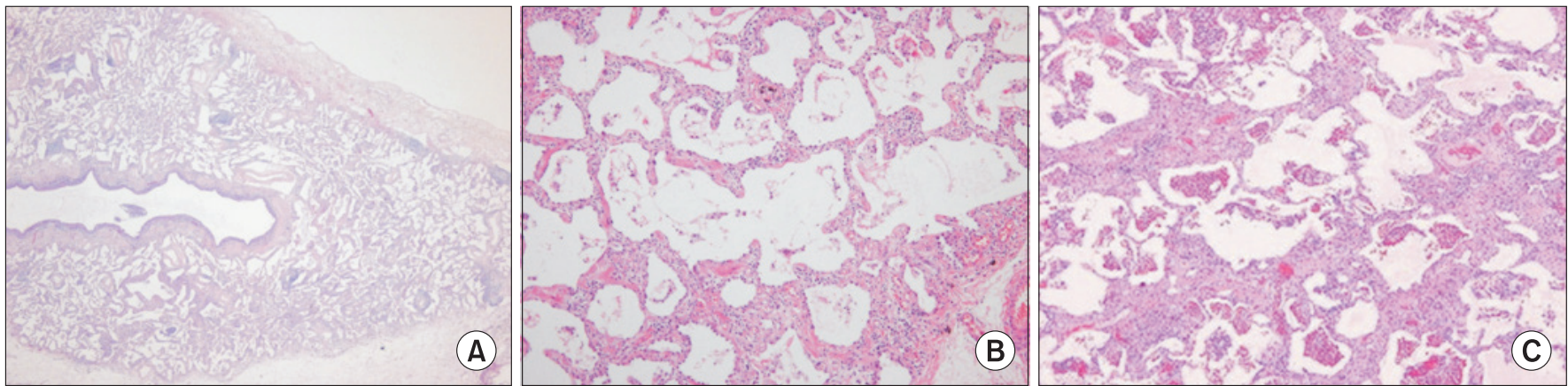

Figure 2. Pathologic findings of nonspecific interstitial pneumonia (NSIP) pattern. (A) Lung architecture is preserved with uniform appearance $(\times 20)$. (B) Cellular NSIP shows uniform thickening of alveolar septa with cellular infiltration $(\times 200)$. (C) Fibrotic NSIP shows uniform thickening of alveolar septa by collagen $(\times 200)$. 
BAL fluid could also be present in HP. If there is no lymphocytosis in the BAL fluid and the fraction of the neutrophil is high, the possibility of IPF rather than NSIP should be considered $^{17,18}$. Transbronchial lung biopsy is not recommended for definite diagnosis of NSIP because the size of specimen is insufficient.

\section{3) Lung biopsy}

Lung biopsy is essential for definite diagnosis of NSIP. The histologic features of NSIP pattern include diffuse interstitial inflammation and fibrosis which are temporally homogeneous. And, usually the basic structure of the alveoli is preserved (Figure 2) ${ }^{2,3}$. NSIP pattern can be classified into cellular and fibrotic NSIP. In cellular NSIP, chronic inflammatory cells infiltrate the alveolar wall and fibrosis of the alveolar wall is hardly observed. Fibrotic NSIP is associated with alveolar wall thickening and fibrosis with or without infiltration of inflammatory cells in the alveolar wall ${ }^{5,19,20}$. Fibrotic NSIP is more common than cellular NSIP and accounts for $80 \%-90 \%$ of the total $\mathrm{NSIP}^{2,21}$. The organizing pneumonia or honeycombing should be sparse or absent. The extent of organizing pneumonia is less than $10 \%$ to $20 \%$, even if observed (Table 2).

The diagnosis of idiopathic NSIP can be summarized as the following.

(1) Clinical symptoms with subacute or chronic course and restrictive ventilatory defect

(2) Radiologic findings which are compatible with NSIP in thoracic HRCT

(3) NSIP pattern in lung biopsy

(4) Exclusion of other causes of NSIP (especially CTD and HP)

\section{Treatment}

\section{PICO for treatment of patients with idiopathic NSIP}

PICO1. Is steroid treatment effective in patients with idiopathic NSIP?

PICO2. Is combination therapy with steroid and immunosuppressive agent more effective than steroid monotherapy in patients with idiopathic NSIP?

\section{1) Recommendations}

- We suggest that clinicians use steroids for treatment of idiopathic NSIP (very low certainty of evidence, strong recommendation).

- We suggest that clinicians use combination with steroids and immunosuppressive agents if steroid monotherapy is ineffective (very low certainty of evidence, weak recom-

Table 2. Histologic features of nonspecific interstitial pneumonia

\begin{tabular}{|c|c|}
\hline & Feature \\
\hline \multicolumn{2}{|c|}{ Critical findings for diagnosis } \\
\hline \multirow[t]{2}{*}{ Cellular pattern } & Mild to moderate interstitial chronic inflammation \\
\hline & Type II pneumocyte hyperplasia in areas of inflammation \\
\hline \multirow[t]{3}{*}{ Fibrosing pattern } & Dense or loose interstitial fibrosis with uniform appearance \\
\hline & Lung architecture is frequently preserved \\
\hline & Interstitial chronic inflammation: mild or moderate \\
\hline \multicolumn{2}{|c|}{ Findings that should consider other diagnosis } \\
\hline \multirow[t]{3}{*}{ Cellular pattern } & Dense interstitial fibrosis: absent \\
\hline & Organizing pneumonia is not the prominent feature (<20\% of biopsy specimen) \\
\hline & Lack of diffuse severe alveolar septal inflammation \\
\hline \multirow[t]{3}{*}{ Fibrosing pattern } & $\begin{array}{l}\text { Temporal heterogeneity pattern: fibroblastic foci with dense fibrosis are inconspicuous or } \\
\text { absent; this is especially important in cases with patchy involvement and subpleural or } \\
\text { paraseptal distribution }\end{array}$ \\
\hline & Honeycombing: inconspicuous or absent \\
\hline & Enlarged fibrotic airspaces may be present \\
\hline \multirow[t]{5}{*}{ Both patterns } & Acute lung injury pattern, especially hyaline membranes: absent \\
\hline & Eosinophils: inconspicuous or absent \\
\hline & Granulomas: absent \\
\hline & Lack of viral inclusions and organisms on special strains for organisms \\
\hline & Dominant airway disease such as extensive peribronchiolar metaplasia \\
\hline
\end{tabular}


mendation).

Idiopathic NSIP is usually treated with steroids and immunosuppressive agents. However, considering the side effects of the mediations, careful observation without treatment can be possible if the disease is mild and meticulous follow up of disease progression with symptoms, pulmonary function and chest HRCT are guaranteed ${ }^{22}$. Because idiopathic NSIP has a low prevalence and has relatively recently been categorized as an independent disease entity, there are no randomized controlled trials that have demonstrated the natural remission rate without treatment or the therapeutic effect of medications. However, previous retrospective studies have reported that steroids and other immunosuppressive agents induce remission or stabilization of symptoms and pulmonary function ${ }^{23,24}$. Kim et $\mathrm{al}^{24}$ reported that among 35 patients with histologically confirmed NSIP who were treated with prednisolone $(0.5 \mathrm{mg} / \mathrm{kg})$, 32 patients survived and pulmonary function improved in 24 patients and maintained stable conditions in 6 patients. In study of Watanabe et al. $^{25}$, after 1 year of steroid treatment, all of 10 patients showed improvement in lung function and oxygenation and only one patient died after 4.3 years of follow-up.

However, this treatment strategy with steroids and immunosuppressive agents has its limitation. It is effective when the inflammatory mechanism is predominant, such as cellular NSIP or organizing pneumonia, but ineffective in patients with fibrotic NSIP ${ }^{26}$. Recently, opinions, that anti-fibrotic drugs used in IPF may be helpful in these fibrotic subtypes, has been raised, but further studies are required ${ }^{22}$.

There is no clear guideline of dose and duration of steroids, but it is recommended that 0.5 to $1.0 \mathrm{mg} / \mathrm{kg}$ or 40 to $60 \mathrm{mg}$ of prednisone can be used as the initial dose. The dose should be maintained for 1 month, and then slowly be reduced ${ }^{23,26}$. If the disease is severe, high-dose methylprednisolone therapy ( $1 \mathrm{~g} /$ day for 3 days, followed by $1 \mathrm{mg} / \mathrm{kg}$ orally, then gradually reduced) may be performed ${ }^{27,28}$.

To make this guideline, the authors attempted a systematic review and meta-analysis of the steroid effect in idiopathic NSIP, but all studies were retrospective and comparative analysis was not possible due to the lack of control group. However, according to Xu et al. ${ }^{29}, 17$ patients (22.9\%) died during the $54 \pm 34$-month follow-up period and 34 patients (45.9\%) died during steroid withdrawal in 74 patients with idiopathic NSIP, which proved the effect of steroids. Park et al. ${ }^{23}$ reported that the mean duration of steroid treatment in NSIP was $17.4 \pm 12.1$ months and $36 \%$ of the patients experienced recurrence. Kim et $\mathrm{al}^{24}$ reported recurrence in six out of 30 steroid-treated patients $(20 \%)$, which was associated with an initial low dose $(0.5$ $\mathrm{mg} / \mathrm{kg}$ ) of steroid and short treatment duration.

Immunosuppressive agents include azathioprine, cyclophosphamide, cyclosporine, and mycophenolate mofetil, which are used for steroid sparing or enhancing the steroid effect. There is no consensus on whether these agents should be initiated at the time of diagnosis or the time of disease pro- gression or the occurrence of steroid dependence. In general, these medications are used when there is a dependence on steroids or the need of steroid maintenance for preventing disease recurrence ${ }^{26,30}$.

Among these immunosuppressive agents, cyclophosphamide is the most frequently used. Kondoh et al. ${ }^{31}$ conducted a study in which they treated 12 histologically confirmed fibrotic NSIP patients with combination of low-dose steroids (20 mg prednisone, every other day) and cyclophosphamide ( 1 to $2 \mathrm{mg} / \mathrm{kg} /$ day). As a results, $33 \%$ showed improvement, $67 \%$ (8/12) showed stable response, and $21 \%$ had side effects related to cyclophosphamide (hemorrhagic cystitis, leukopenia, myelodysplastic syndrome, and infection) $)^{31}$. Corte et $\mathrm{al}^{32}$ reviewed 54 patients with rapid progression of NSIP who were treated with IV cyclophosphamide $\left(600 \mathrm{mg} / \mathrm{m}^{2}\right)$ and observed good response in most of the patients after 6 months of treatment. Effects of other drugs such as azathioprine, cyclosporine, and mycophenolate mofetil have been reported in a small number of cases ${ }^{4}$.

On the other hand, literature review was conducted to confirm whether the combination of steroids and immunosuppressive agents is more effective than steroid monotherapy. In a study published by Fujita et al. ${ }^{10}, 22$ patients with idiopathic NSIP were treated (19 with steroids and 3 with steroids and cyclophosphamide), and the mortality rate of patients with combination therapy was 1.5 times (95\% confidence interval, 0.26-9.79) that of the patients with steroid monotherapy. However, the study was a small, retrospective study and the patients who were more severe or unresponsive to steroids may have treated with combination therapy. Therefore, the results of this study were needed to be interpreted with caution. Recently, Keir et al. ${ }^{33}$ reported the hopeful results with rituximab in 50 patients with severe interstitial lung disease who did not respond to conventional immunosuppressive agents except IPF. In the future, studies on the efficacy of these drugs in idiopathic NSIP need to be conducted.

There is no standardized method for assessing therapeutic response in idiopathic NSIP. However, like IPF, treatment response can be measured by combining the changes of HRCT, symptoms such as dyspnea or cough and pulmonary function test (forced vital capacity [FVC] and carbon monoxide diffusion capacity [DLco]), usually between 3 and 6 months after the start of treatment ${ }^{34}$. Changes in pulmonary function over time are known to be useful surrogate marker of survival. Reduction of DLco over $15 \%$ after 12 months or decrease of FVC over 10\% between 6 and 12 months are independently associated with mortality ${ }^{23,35,36}$. Therefore, when DLco is reduced over $15 \%$ or FVC is reduced over $10 \%$ despite treatment without any specific reason such as infection, other treatments such as changing medications or lung transplantation should be considered ${ }^{37}$. In addition to pharmacologic treatment, oxygen therapy for hypoxemia at night or during exercise, treatment for concomitant diseases such as reflux esophagitis 
or pulmonary hypertension, and symptomatic therapy for dyspnea or cough can be performed ${ }^{38}$.

\section{Natural Course and Prognosis}

Survival of NSIP is better than $\operatorname{IPF}^{39}$. Many studies have reported a 5 -year survival rate of more than $70 \%$, and particularly in the case of cellular NSIP, there are few disease-related deaths ${ }^{2,723}$. Treatment of idiopathic NSIP, although not well proven, is generally begin with steroid only or combined with immunosuppressants such as azathioprine, cyclophosphamide, cyclosporine, and mycophenolate mofetil. The clinical response after the first treatment was generally favorable. About two thirds of patients have improved after initial treatment.

However, frequent recurrences were reported after discontinuation of treatment. Kim et al. ${ }^{24}$ reported recurrence in $20 \%$ and Park et al. ${ }^{23}$ reported recurrence in $36 \%$, and the prognosis of patients with recurrence was worse. In addition, as in the IPF, there may be acute exacerbation during the course of NSIP, and Park et al. ${ }^{40}$ reported acute exacerbation of $4.2 \%$ for one year in patients with idiopathic NSIP.

NSIP pattern is the most common interstitial pneumonia that occurs in CTDs. Therefore, it has always been suggested that NSIP may be the first or the only manifestation of CTD. The possibility that NSIP could be the first manifestation of CTD was firstly raised by Sato et al. ${ }^{41}$. Kinder et al. ${ }^{11}$ reported that $80 \%$ of 28 idiopathic NSIPs met the criteria for undifferentiated connective tissue disease (UCTD), and Park et al. ${ }^{23}$ reported that eight of 83 patients (10\%) initially thought to be idiopathic NSIP were diagnosed with CTD in the process of their illness. In fact, a large number of patients with NSIP currently do not meet the criteria for specific CTD have some features of CTD. The researchers described these patients with other names, such as UCTD-interstitial lung disease, lung-dominant CTD, or autoimmune featured ILD. Recently, ATS/ERS proposed "interstitial pneumonia with autoimmune features (IPAF)" for them ${ }^{42}$. Therefore, patients with histologically NSIP pattern should go through sufficient consideration of the presence of CTD, and the occurrence of CTD should be carefully observed in the following process.

\section{Authors' Contributions}

Conceptualization: Kim YH. Methodology: Kang JY. Formal analysis: Jegal Y. Data curation: Park SY. Software: Lee J. Validation: Kim YH. Investigation: Lee J, Kim YH. Writing - original draft preparation: Lee J. Writing - review and editing: Kim YH, Kang JY, Jegal Y, Park SY. Approval of final manuscript: all authors.

\section{Conflicts of Interests}

No potential conflict of interest relevant to this article was reported.

\section{Funding}

No funding to declare.

\section{References}

1. Travis WD, Costabel U, Hansell DM, King TE Jr, Lynch DA, Nicholson AG, et al. An official American Thoracic Society/ European Respiratory Society statement: update of the international multidisciplinary classification of the idiopathic interstitial pneumonias. Am J Respir Crit Care Med 2013;188:733-48.

2. Travis WD, Hunninghake G, King TE Jr, Lynch DA, Colby TV, Galvin JR, et al. Idiopathic nonspecific interstitial pneumonia: report of an American Thoracic Society project. Am J Respir Crit Care Med 2008;177:1338-47.

3. Katzenstein AL, Fiorelli RF. Nonspecific interstitial pneumonia/fibrosis: histologic features and clinical significance. Am J Surg Pathol 1994;18:136-47.

4. American Thoracic Society; European Respiratory Society. American Thoracic Society/European Respiratory Society International Multidisciplinary Consensus Classification of the Idiopathic Interstitial Pneumonias. This joint statement of the American Thoracic Society (ATS), and the European Respiratory Society (ERS) was adopted by the ATS board of directors, June 2001 and by the ERS Executive Committee, June 2001. Am J Respir Crit Care Med 2002;165:277-304.

5. Flaherty KR, Martinez FJ. Nonspecific interstitial pneumonia. Semin Respir Crit Care Med 2006;27:652-8.

6. Hyldgaard C, Hilberg O, Muller A, Bendstrup E. A cohort study of interstitial lung diseases in central Denmark. Respir Med 2014;108:793-9.

7. Scientific Committee of the Korean Academy of Tuberculosis and Respiratory Diseases. 2008 National survey of idiopathic interstitial pneumonia in Korea. Tuberc Respir Dis 2009;66: 141-51.

8. Raghu G, Remy-Jardin M, Myers JL, Richeldi L, Ryerson CJ, Lederer DJ, et al. Diagnosis of idiopathic pulmonary fibrosis. An Official ATS/ERS/JRS/ALAT Clinical Practice Guideline. Am J Respir Crit Care Med 2018;198:e44-68.

9. Romagnoli M, Nannini C, Piciucchi S, Girelli F, Gurioli C, Casoni G, et al. Idiopathic nonspecific interstitial pneumonia: an interstitial lung disease associated with autoimmune disorders? Eur Respir J 2011;38:384-91.

10. Fujita J, Ohtsuki Y, Yoshinouchi T, Yamadori I, Bandoh S, Tokuda M, et al. Idiopathic non-specific interstitial pneumo- 
nia: as an "autoimmune interstitial pneumonia". Respir Med 2005;99:234-40.

11. Kinder BW, Collard HR, Koth L, Daikh DI, Wolters PJ, Elicker $\mathrm{B}$, et al. Idiopathic nonspecific interstitial pneumonia: lung manifestation of undifferentiated connective tissue disease? Am J Respir Crit Care Med 2007;176:691-7.

12. Kim DS, Yoo B, Lee JS, Kim EK, Lim CM, Lee SD, et al. The major histopathologic pattern of pulmonary fibrosis in scleroderma is nonspecific interstitial pneumonia. Sarcoidosis Vasc Diffuse Lung Dis 2002;19:121-7.

13. Collins B, Raghu G. Interstitial pneumonia with autoimmune features: the new consensus-based definition for this cohort of patients should be broadened. Eur Respir J 2016;47:1293-5.

14. Silva CI, Muller NL, Hansell DM, Lee KS, Nicholson AG, Wells AU. Nonspecific interstitial pneumonia and idiopathic pulmonary fibrosis: changes in pattern and distribution of disease over time. Radiology 2008;247:251-9.

15. Hartman TE, Swensen SJ, Hansell DM, Colby TV, Myers JL, Tazelaar HD, et al. Nonspecific interstitial pneumonia: variable appearance at high-resolution chest CT. Radiology 2000; 217:701-5.

16. Bna C, Zompatori M, Poletti V, Spaggiari E, Chetta A, Calabro E, et al. Differential diagnosis between usual interstitial pneumonia (UIP) and nonspecific interstitial pneumonia (NSIP) assessed by high-resolution computed tomography (HRCT). Radiol Med 2005;109:472-87.

17. Ryu YJ, Chung MP, Han J, Kim TS, Lee KS, Chun EM, et al. Bronchoalveolar lavage in fibrotic idiopathic interstitial pneumonias. Respir Med 2007;101:655-60.

18. Veeraraghavan S, Latsi PI, Wells AU, Pantelidis P, Nicholson AG, Colby TV, et al. BAL findings in idiopathic nonspecific interstitial pneumonia and usual interstitial pneumonia. Eur Respir J 2003;22:239-44.

19. Leslie KO. Historical perspective: a pathologic approach to the classification of idiopathic interstitial pneumonias. Chest 2005;128(5 Suppl 1):513S-9S.

20. Nicholson AG, Colby TV, du Bois RM, Hansell DM, Wells AU. The prognostic significance of the histologic pattern of interstitial pneumonia in patients presenting with the clinical entity of cryptogenic fibrosing alveolitis. Am J Respir Crit Care Med 2000;162:2213-7.

21. Kligerman SJ, Groshong S, Brown KK, Lynch DA. Nonspecific interstitial pneumonia: radiologic, clinical, and pathologic considerations. Radiographics 2009;29:73-87.

22. Wells AU, Kokosi M, Karagiannis K. Treatment strategies for idiopathic interstitial pneumonias. Curr Opin Pulm Med 2014;20:442-8.

23. Park IN, Jegal Y, Kim DS, Do KH, Yoo B, Shim TS, et al. Clinical course and lung function change of idiopathic nonspecific interstitial pneumonia. Eur Respir J 2009;33:68-76.

24. Kim YJ, Park JW, Kyung SY, Lee SP, Chung MP, Kim YH, et al. Clinical characteristics of idiopathic pulmonary fibrosis patients with diabetes mellitus: the national survey in Korea from 2003 to 2007. J Korean Med Sci 2012;27:756-60.

25. Watanabe K, Higuchi K, Ninomiya K, Ohshima T, Aritomi T, Kawabata Y, et al. Steroid treatment based on the findings of transbronchial biopsy in idiopathic interstitial pneumonia. Eur Respir J 2002;20:1213-9.

26. Tomassetti S, Ryu JH, Piciucchi S, Chilosi M, Poletti V. Nonspecific interstitial pneumonia: what is the optimal approach to management? Semin Respir Crit Care Med 2016;37:37894.

27. Flaherty KR, Toews GB, Travis WD, Colby TV, Kazerooni EA, Gross BH, et al. Clinical significance of histological classification of idiopathic interstitial pneumonia. Eur Respir J 2002;19:275-83.

28. Nanki N, Fujita J, Yamaji Y, Maeda H, Kurose T, Kaji M, et al. Nonspecific interstitial pneumonia/fibrosis completely recovered by adding cyclophosphamide to corticosteroids. Intern Med 2002;41:867-70.

29. Xu W, Xiao Y, Liu H, Qin M, Zheng W, Shi J. Nonspecific interstitial pneumonia: clinical associations and outcomes. BMC Pulm Med 2014;14:175.

30. Kinder BW. Nonspecific interstitial pneumonia. Clin Chest Med 2012;33:111-21.

31. Kondoh Y, Taniguchi H, Yokoi T, Nishiyama O, Ohishi T, Kato $\mathrm{T}$, et al. Cyclophosphamide and low-dose prednisolone in idiopathic pulmonary fibrosis and fibrosing nonspecific interstitial pneumonia. Eur Respir J 2005;25:528-33.

32. Corte TJ, Ellis R, Renzoni EA, Hansell DM, Nicholson AG, du Bois RM, et al. Use of intravenous cyclophosphamide in known or suspected, advanced non-specific interstitial pneumonia. Sarcoidosis Vasc Diffuse Lung Dis 2009;26:132-8.

33. Keir GJ, Maher TM, Ming D, Abdullah R, de Lauretis A, Wickremasinghe $\mathrm{M}$, et al. Rituximab in severe, treatment-refractory interstitial lung disease. Respirology 2014;19:353-9.

34. Poletti V, Romagnoli M, Piciucchi S, Chilosi M. Current status of idiopathic nonspecific interstitial pneumonia. Semin Respir Crit Care Med 2012;33:440-9.

35. Latsi PI, du Bois RM, Nicholson AG, Colby TV, Bisirtzoglou D, Nikolakopoulou A, et al. Fibrotic idiopathic interstitial pneumonia: the prognostic value of longitudinal functional trends. Am J Respir Crit Care Med 2003;168:531-7.

36. Jegal Y, Kim DS, Shim TS, Lim CM, Lee SD, Koh Y, et al. Physiology is a stronger predictor of survival than pathology in fibrotic interstitial pneumonia. Am J Respir Crit Care Med 2005;171:639-44.

37. Belloli EA, Beckford R, Hadley R, Flaherty KR. Idiopathic nonspecific interstitial pneumonia. Respirology 2016;21:259-68.

38. Troy L, Corte TJ. Management of the idiopathic interstitial pneumonias. Aust Prescr 2012;35:202-6.

39. Bjoraker JA, Ryu JH, Edwin MK, Myers JL, Tazelaar HD, Schroeder DR, et al. Prognostic significance of histopathologic subsets in idiopathic pulmonary fibrosis. Am J Respir Crit Care Med 1998;157:199-203.

40. Park IN, Kim DS, Shim TS, Lim CM, Lee SD, Koh Y, et al. Acute 
exacerbation of interstitial pneumonia other than idiopathic pulmonary fibrosis. Chest 2007;132:214-20.

41. Sato T, Fujita J, Yamadori I, Ohtsuki Y, Yoshinouchi T, Bandoh $\mathrm{S}$, et al. Non-specific interstitial pneumonia; as the first clinical presentation of various collagen vascular disorders. Rheumatol Int 2006;26:551-5.
42. Fischer A, Antoniou KM, Brown KK, Cadranel J, Corte TJ, du Bois RM, et al. An official European Respiratory Society/ American Thoracic Society research statement: interstitial pneumonia with autoimmune features. Eur Respir J 2015;46: 976-87. 